\title{
Incidencia de falla anastomótica en intestino delgado, colon y recto, Bogotá, Colombia
}

\author{
Maikel Adolfo Pacheco ${ }^{1}$, Guillermo Eduardo Aldana ${ }^{2}$, Luis Eduardo Martínez ${ }^{3}$, Juan Carlos Forero 4 , \\ Carlos Andrés Gómez 5 , Esteban Mauricio Coral ${ }^{4}$, Héctor Guillermo Olaya ${ }^{4}$
}

Palabras clave: intestino delgado; técnicas de sutura; anastomosis quirúrgica; dehiscencia de la herida operatoria; fístula intestinal; desnutrición proteico-calórica.

\section{Resumen}

Introducción. La falla anastomótica es una de las principales complicaciones de las cirugías gastrointestinales, que se presenta en 3,4 a $15 \%$ de los pacientes, aproximadamente. En Colombia, no se conoce con exactitud la incidencia de falla anastomótica.

1 Profesor asociado, Departamento de Cirugía General, Fundación Universitaria de Ciencias de la Salud-Hospital de San José, Hospital Infantil Universitario de San José de Bogotá, Bogotá D.C., Colombia

2 Cirujano hepatobiliar y de trasplante renal; vicepresidente, Asociación Colombiana de Cirugía Hepatobiliar; cirujano adscrito, Hospital Universitario Infantil de San José de Bogotá, Bogotá D.C., Colombia

3 Instructor asistente, Departamento de Cirugía General, Fundación Universitaria de Ciencias de la Salud-Hospital de San José de Bogotá, Bogotá D.C., Colombia

4 Residente de cuarto año de Cirugía General, Fundación Universitaria de Ciencias de la Salud-Hospital de San José, Hospital Infantil Universitario de San José de Bogotá, Bogotá D.C., Colombia

5 Residente de tercer año de Cirugía General, Fundación Universitaria de Ciencias de la Salud-Hospital de San José, Hospital Infantil Universitario de San José de Bogotá, Bogotá D.C., Colombia

Fecha de recibido: 18 de mayo de 2017

Fecha de aprobación: 8 de septiembre de 2017

Citar como: Pacheco MA, Aldana GE, Martínez LE, Forero JC, Gómez CA, Coral EM, et al. Incidencia de falla anastomótica en intestino delgado, colon y recto, Bogotá, Colombia. Rev Colomb Cir. 2017;32:269-76.
Objetivo. Determinar la incidencia de falla anastomótica en cirugias intestinales en dos hospitales de IV nivel de Bogotá.

Métodos. Se llevó a cabo un estudio prospectivo de cohorte que incluyó pacientes sometidos a anastamosis y a quienes se les hizo seguimiento hasta de 30 dias. Se realizó un análisis descriptivo de la información, asociaciones entre variables mediante la prueba de ji al cuadrado y, además, se emplearon gráficas de KaplanMeier para determinar el tiempo hasta el evento. Los datos se analizaron en Stata $13^{\circledR}$.

Resultados. Se incluyeron 195 pacientes. El 47,1\% era de sexo masculino y la mediana de la edad fue de 60 años (rango intercuartílico, RIC: 48 a 70). La incidencia de falla anastomótica fue de 10,8\% y la tasa de incidencia fue de 10,2 por 1.000 personas/año. El 29,7\% de los pacientes presentaron complicaciones posoperatorias y se presentó una mortalidad de 9,7\%. La mediana de la estancia hospitalaria fue de 8 dias (RIC: 5 a 16).

Conclusiones. La incidencia de fistula de la anastomosis que se presentó estuvo acorde con lo reportado en la literatura científica a nivel mundial. El antecedente de cirugía abdominal así como la cirugía de urgencia fueron los factores frecuentes entre los pacientes que presentaron falla anastomótica.

\section{Introducción}

Dentro del contexto histórico sobre el advenimiento de las anastomosis intestinales, surge como principal exponente Lembert, quien desarrolló una técnica de sutura intesti- 
nal afrontando capas de serosa, con buenos resultados. Sin embargo, fue Theodor Billroth quien, utilizando conceptos de Lembert, realizó la primera anastomosis exitosa en $1881^{1,2}$. Desde entonces, han pasado más de cien años y los avances en los dispositivos y las técnicas han permitido disminuir las complicaciones y los tiempos quirúrgicos; las suturas mecánicas en el campo de la cirugía fueron introducidas por los húngaros Hult y Fisher por primera vez en $1908^{1}$.

La fístula gastrointestinal, o falla anastomótica, fue definida por Berry y Fisher en 1996, como "toda comunicación entre dos superficies intestinales epitelizadas (sic) después de una anastomosis intestinal que ocasiona manifestaciones clínicas características luego de la exposición de la cavidad peritoneal a material intestinal" " ${ }^{3}$. Según este concepto, las fístulas se pueden clasificar según su ubicación anatómica o su mecanismo fisiológico, este último, determinado por su producción en volumen diario (tabla 1) ${ }^{4,5}$. En contraste con las fístulas de intestino delgado, las de colon y recto son poco frecuentes, y pueden ser externas o internas con relación varios órganos. El proceso patológico más frecuente implicado en el desarrollo espontáneo de las fístulas del colon, es la enfermedad diverticular complicada. Es menos común encontrar fístulas causadas por la enfermedad de Crohn o por enfermedad maligna ${ }^{6,7}$. Entre estas fistulas del colon, el porcentaje global puede fluctuar entre el 3,4 y el 6,0 \% ${ }^{3,4,8,9}$.

Los criterios clínicos para el diagnóstico de la fístula pueden incluir signos de reacción inflamatoria, salida de

TABLA 1.

Clasificación de fístulas gastrointestinales

\begin{tabular}{ll}
\hline \multicolumn{1}{c}{ Mecanismo } & \multicolumn{1}{c}{ Características } \\
\hline \multirow{2}{*}{ Ubicación anatómica } & $\begin{array}{l}\text { Estómago } \\
\text { Intestino delgado } \\
\text { Intestino grueso }\end{array}$ \\
\hline \multirow{2}{*}{$\begin{array}{l}\text { Mecanismo físiológico } \\
\text { (volumen) }\end{array}$} & Bajo gasto: $<200 \mathrm{ml} /$ día \\
& Alto gasto: $>500 \mathrm{ml} /$ día \\
\hline & Iatrogénico \\
& Trauma \\
& Cuerpo extraño \\
& Enfermedad inflamatoria \\
Etiología & Infección \\
& Neoplasia maligna \\
\hline
\end{tabular}

material intestinal por la herida y deterioro hemodinámico. Una vez instaurado cualquiera de estos signos o síntomas, se requiere del apoyo de exámenes de laboratorio, en los cuales es muy común encontrar leucocitosis, neutrofilia, elevación de azoados y alteración de la función hepática. El diagnóstico se confirma al comprobar la filtración de contenido intestinal; comúnmente, esto se observa en la tomografía axial con contraste, la cual presenta sensibilidad y especificidad hasta del $95 \%$. Sin embargo, el método estándar para el diagnóstico de fístula sigue siendo la exploración quirúrgica, la cual permite no solo identificar la presencia de fístula, sino que también brinda la posibilidad de un tratamiento definitivo ${ }^{10-13}$.

Las fugas de la anastomosis, generalmente, ocurren durante los primeros siete días posteriores a la cirugía, con una instauración rápida de infección local con posterior diseminación hematógena, lo cual lleva a un choque séptico de difícil manejo; si se presenta falla orgánica múltiple, es posible que el índice de mortalidad se incremente llegando hasta el $80 \%{ }^{9}$.

Al revisar la literatura científica nacional, no se encontraron registros de la incidencia de falla anastomótica y debido a que no hay una herramienta útil estandarizada y del todo confiable que ayude a disminuir las fallas en las anastomosis, es importante que cada institución conozca el número de casos y el número de complicaciones y, así, determine en cuál de estas se pudo haber intervenido favorablemente para evitarlas. Por esta razón, en este estudio se pretende determinar la incidencia de falla anastomótica intestinal en dos hospitales de IV nivel de Bogotá.

\section{Métodos}

Se llevó a cabo un estudio prospectivo de cohorte que incluyó pacientes mayores de 18 años, sometidos a anastomosis intestinal (intestino delgado, colon o recto) por el Servicio de Cirugía General. Se excluyeron aquellos pacientes sometidos a anastomosis biliopancreáticas o gastrointestinales.

El tamaño de la muestra se calculó considerando una incidencia del $15 \%$ (valor máximo reportado en la literatura) ${ }^{3,5}$, un nivel de confianza del $95 \%$ y un error del $5 \%$, con lo cual el resultado fue de 195 pacientes incluidos. 
Los datos se registraron diariamente por medio de un formato único de recolección, utilizando la décima actualización de la Clasificación Internacional de Enfermedades (CIE-10). Se hizo seguimiento a los 30 días del procedimiento mediante control posoperatorio ambulatorio, el cual fue asignado en el momento del egreso hospitalario. El principal parámetro evaluado fue la incidencia cruda; para su cálculo, en el denominador se consideró el total de pacientes incluidos en el estudio. Otras variables evaluadas fueron las características demográficas de la población, la estancia hospitalaria, las complicaciones posoperatorias diferentes a la falla en la anastomosis, así como la técnica y los materiales usados en la intervención quirúrgica.

Se hizo un análisis descriptivo de las variables; las variables cualitativas se muestran como frecuencias absolutas y relativas, y las variables cuantitativas, como medianas y rangos intercuartílicos dado que los datos no eran de distribución normal, lo cual fue evaluado mediante la prueba de Shapiro-Wilk. Se hicieron asociaciones entre variables mediante la prueba de ji al cuadrado o el test exacto de Fisher, según la distribución de las celdas de la tabla de contingencia; un valor de $\mathrm{p}$ inferior a 0,05 se consideró como estadísticamente significativo. También, se utilizaron las curvas de Kaplan-Meier para determinar el tiempo de estancia hospitalaria hasta la fuga anastomótica. Los análisis se llevaron a cabo con el software estadístico Stata $13^{\circledR}$.

\section{Resultados}

Durante el periodo comprendido entre febrero de 2016 y febrero de 2017, se recolectaron 195 pacientes y no se presentaron pérdidas durante el seguimiento. Del total de pacientes, 21 presentaron filtración de la anastomosis, lo que equivale a una incidencia del $10,7 \%$. Se obtuvo una mediana de edad de 60 años (rango intercuartílico, RIC: 48 a 70); el 47,1 \% de los pacientes era de sexo masculino; el sobrepeso y el antecedente de cirugía abdominal se presentaron con mayor prevalencia tanto en los pacientes que presentaron la fuga como en los que no la presentaron (tabla 2).

El 54,9\% del total de pacientes presentó un estado nutricional normal y tan solo $5,1 \%$ tenía un estado grave; se encontraron diferencias estadísticamente significativas entre el estado nutricional según la presencia
TABLA 2.

Características generales de la población

\begin{tabular}{|c|c|c|c|}
\hline Características & $\begin{array}{c}\text { Fístulas }(\mathrm{n}=\mathbf{2 1}) \\
\mathrm{n}(\%)\end{array}$ & $\begin{array}{c}\text { Sin fístulas } \\
(\mathrm{n}=174) \\
\mathrm{n}(\%)\end{array}$ & $\begin{array}{c}\text { Total }(n=195) \\
\text { n }(\%)\end{array}$ \\
\hline $\begin{array}{l}\text { Edad en años - } \\
\text { Media (RIC) }\end{array}$ & $68,0(46,0-74,0)$ & $49,0(59,5-69,0)$ & $60,0(48,0-70,0)$ \\
\hline Sexo masculino & 11 & 81 & 92 \\
\hline \multicolumn{4}{|c|}{ Índice de masa corporal } \\
\hline Normal & $9(42,9)$ & $69(39,7)$ & $78(40,0)$ \\
\hline Sobrepeso & $9(42,9)$ & $80(46,0)$ & $89(45,6)$ \\
\hline $\begin{array}{l}\text { Obesidad } \\
\text { Grado I }\end{array}$ & $0(0,0)$ & $21(2,3)$ & $21(10,8)$ \\
\hline Grado II & $0(0,0)$ & $4(2,3)$ & $4(2,1)$ \\
\hline Grado III & $3(14,3)$ & $0(0,0)$ & $3(1,5)$ \\
\hline \multicolumn{4}{|l|}{ Antecedentes } \\
\hline $\begin{array}{l}\text { Hipertensión } \\
\text { arterial }\end{array}$ & $7(33,3)$ & $49(28,2)$ & $56(28,7)$ \\
\hline $\begin{array}{l}\text { Diabetes } \\
\text { mellitus }\end{array}$ & $3(14,3)$ & $23(13,2)$ & $26(13,3)$ \\
\hline Tabaquismo & $3(14,3)$ & $19(10,9)$ & $22(11,3)$ \\
\hline $\begin{array}{l}\text { Enfermedad } \\
\text { cardiovascular }\end{array}$ & $0(0,0)$ & $15(8,6)$ & $15(7,7)$ \\
\hline Desnutrición & $1(4,8)$ & $5(2,9)$ & $6(3,1)$ \\
\hline $\begin{array}{l}\text { Cirugía } \\
\text { abdominal }\end{array}$ & $16(76,2)$ & $109(62,6)$ & $125(64,1)$ \\
\hline
\end{tabular}

o ausencia de filtración ( $\mathrm{p}=0,037)$. Además, se encontró que la oportunidad de la cirugía (electiva de urgencia), el material de sutura y la experiencia del cirujano que practicó el procedimiento, eran factores asociados a la falla anastomótica (tabla 3 ).

En el seguimiento posoperatorio, se documentó que $58(29,7 \%)$ pacientes presentaron complicaciones posoperatorios, 38 de las cuales fueron infección del sitio operatorio: cuatro superficiales, una profunda, y 33 de órgano y espacio. La mortalidad total fue de 9,7\%: 10 de $21(47,6 \%)$ casos con falla anastomótica y 9 de 174 $(5,2 \%)$ casos sin falla anastomótica (tabla 3$)$.

La mediana de tiempo transcurrido hasta la presencia de la falla anastomótica fue de 7 días (RIC: 5 a 8) (figura 1). La mediana de la estancia hospitalaria fue de 8 días (RIC: 5 a 16) (figura 2); la estancia hospitalaria fue mayor en los pacientes que presentaron filtración (figura 3 ). 
TABLA 3.

Características clínicas de la población

\begin{tabular}{|c|c|c|c|c|}
\hline Características & $\begin{array}{c}\text { Total } \\
(\mathrm{N}=195) \\
\text { n }(\%)\end{array}$ & $\begin{array}{c}\text { Fístula } \\
(n=21) \\
n(\%)\end{array}$ & $\begin{array}{c}\text { Sin fístulas } \\
(\mathrm{n}=174) \\
\text { n }(\%)\end{array}$ & $\mathbf{p}$ \\
\hline \multicolumn{5}{|l|}{ Estado nutricional } \\
\hline Normal & $107(54,9)$ & $7(33,3)$ & $100(57,5)$ & \multirow{4}{*}{$0,037 *$} \\
\hline Leve & $52(26,7)$ & $6(28,6)$ & $46(26,4)$ & \\
\hline Moderado & $26(13,3)$ & $5(23,8)$ & $21(12,1)$ & \\
\hline Grave & $10(5,1)$ & $3(14,3)$ & $7(4,0)$ & \\
\hline \multicolumn{5}{|l|}{ Oportunidad } \\
\hline Cirugía electiva & $102(52,3)$ & $4(19,0)$ & $98(56,3)$ & \multirow[t]{2}{*}{$0,002 *$} \\
\hline Cirugía de urgencia & $93(47,7)$ & $17(81,0)$ & $76(43,7)$ & \\
\hline \multicolumn{5}{|l|}{ Tipo de anastomosis } \\
\hline Término-terminal & $98(50,3)$ & $8(38,1)$ & $90(51,7)$ & \multirow{3}{*}{0,422} \\
\hline Latero-lateral & $79(40,5)$ & $10(47,6)$ & $69(39,7)$ & \\
\hline Término-lateral & $18(9,2)$ & $3(14,3)$ & $15(8,6)$ & \\
\hline \multicolumn{5}{|c|}{ Ubicación de la anastomosis intestinal } \\
\hline Delgado-delgado & $79(40,5)$ & $10(47,6)$ & $69(39,7)$ & \multirow{4}{*}{0,544} \\
\hline Delgado-grueso & $53(27,2)$ & $7(33,3)$ & $46(26,4)$ & \\
\hline Grueso-grueso & $22(11,3)$ & $2(9,5)$ & $20(11,5)$ & \\
\hline Grueso-recto & $41(21,0)$ & $2(9,5)$ & $39(22,4)$ & \\
\hline \multicolumn{5}{|l|}{ Material de sutura $(n=152)$} \\
\hline Polipropileno & $75(49,3)$ & $8(44,4)$ & $67(50,0)$ & \multirow{4}{*}{$0,017 *$} \\
\hline Ácido poliglicólico & $65(42,8)$ & $7(38,9)$ & $58(43,3)$ & \\
\hline Polidioxanona & $8(5,3)$ & $0(0,0)$ & $8(6,0)$ & \\
\hline Seda & $4(2,6)$ & $3(16,7)$ & & \\
\hline \multicolumn{4}{|l|}{ Sangrado } & \multirow[t]{4}{*}{0,819} \\
\hline$<200$ & $136(69,7)$ & $14(66,7)$ & $122(70,1)$ & \\
\hline $200-400$ & $34(17,4)$ & $4(19,0)$ & $30(17,2)$ & \\
\hline$>400$ & $25(12,8)$ & $3(14,3)$ & $22(12,6)$ & \\
\hline \multicolumn{4}{|l|}{ Experiencia del cirujano } & \multirow[t]{6}{*}{$0,004 *$} \\
\hline $\begin{array}{l}\text { Residente de cirugía } \\
\text { general }\end{array}$ & $49(25,1)$ & $8(38,1)$ & $41(23,6)$ & \\
\hline $\begin{array}{l}\text { Instructor menos de } \\
1 \text { año }\end{array}$ & $3(1,5)$ & $1(4,8)$ & $2(1,1)$ & \\
\hline $\begin{array}{l}\text { Instructor entre } 1 \text { y } \\
5 \text { años }\end{array}$ & $20(10,3)$ & $4(19,0)$ & $16(9,2)$ & \\
\hline $\begin{array}{l}\text { Instructor entre } 5 \text { y } \\
10 \text { años }\end{array}$ & $18(9,2)$ & $4(19,0)$ & $14(8,0)$ & \\
\hline $\begin{array}{l}\text { Instructor más de } 10 \\
\text { años }\end{array}$ & $105(53,8)$ & $4(19,0)$ & $101(58,0)$ & \\
\hline $\begin{array}{l}\text { Complicaciones } \\
\text { posoperatorias }\end{array}$ & $58(29,7)$ & $20(95,2)$ & $38(21,8)$ & - \\
\hline $\begin{array}{l}\text { Estancia hospitalaria - } \\
\text { Media (RIC) }\end{array}$ & $8(5$ a 16$)$ & $\begin{array}{c}25(14 \mathrm{a} \\
31)\end{array}$ & $7(5$ a 13$)$ & - \\
\hline Muerte & $19(9,7)$ & $10(47,6)$ & $9(5,2)$ & - \\
\hline
\end{tabular}

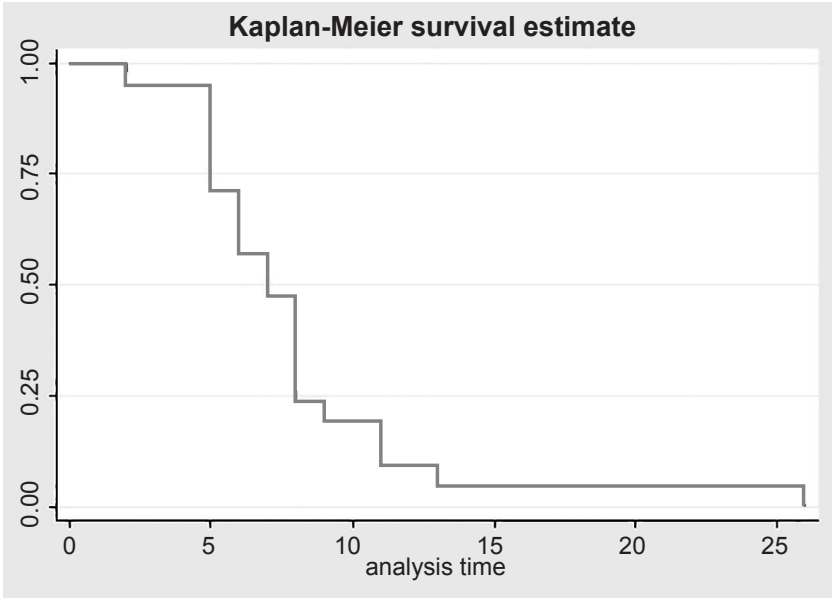

Figura 1. Tiempo hasta la aparición de fuga anastomótica

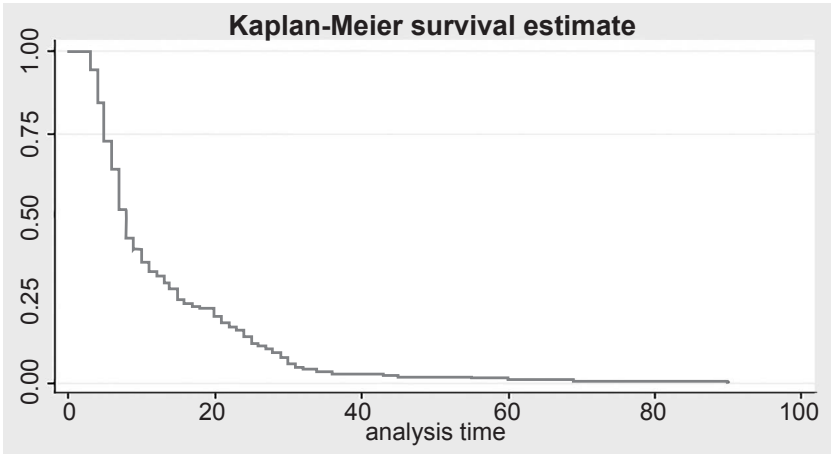

Figura 2. Tiempo de estancia hospitalaria

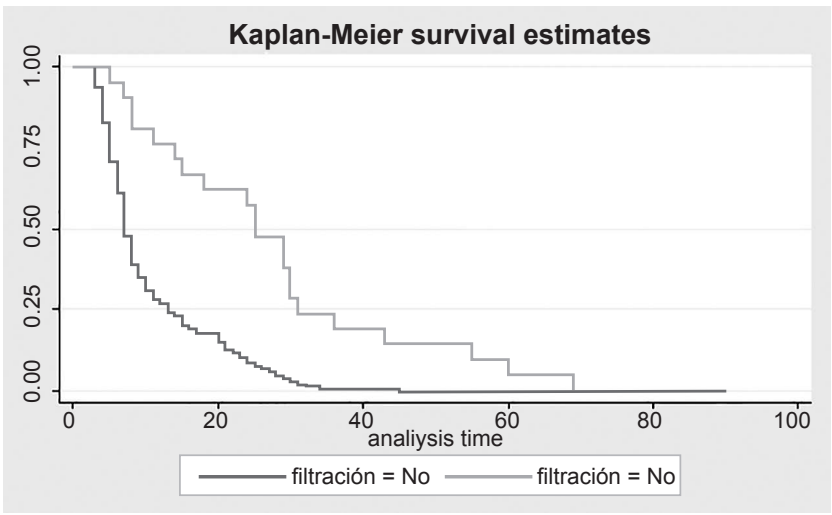

FiguRA 3. Tiempo de estancia hospitalaria en pacientes según presencia de falla anastomótica

\section{Discusión}

La fístula en la anastomosis es una de las principales complicaciones de las cirugías gastrointestinales, con una incidencia que oscila entre 3,4 y $15 \%$, datos que varían según el segmento gastrointestinal anastomosado, así 
como múltiples factores propios del paciente, el medio y el cirujano ${ }^{3,5,6,14}$.

Por lo anterior, se determinó la incidencia de falla en la anastomosis, medida por la presencia de fístula intestinal, en dos instituciones de cuarto nivel de Bogotá. Se encontró que $10,7 \%$ de los pacientes presentaron fístulas de anastomosis, porcentaje estimado y esperado dada la complejidad y el número de casos en el periodo evaluado.

En la literatura científica mundial se informa un valor máximo de $15 \%$ para estas complicaciones. Sin embargo, son muchas las variables y las estimaciones que en este tipo de estudios se deben tener en cuenta al hacer el análisis estadístico. Tal vez uno de los aportes más representativos en este campo fue el Byrn, et al., en 2006, en un estudio retrospectivo de 1.684 pacientes sometidos a resección intestinal, 38 de los cuales presentaron fístula de la anastomosis, lo cual demostró que esta complicación sigue presentándose en este tipo de intervención quirúrgica ${ }^{15}$.

En el presente estudio, la mediana de edad fue de 60 años, con ligero predominio del sexo femenino $(52,8 \%)$, cifras que no están muy lejanas de las informadas a nivel mundial. Según Campos, et al., la edad del paciente influye mucho en la mortalidad secundaria a fístula, pero no en su cierre primario ${ }^{16}$; en el estudio de Levy, et al. (1989), una edad de más de 50 años se consideró como factor predictor de fístula intestinal ${ }^{17}$.

Por otra parte, Lipska, et al., determinaron que el sexo masculino era un factor de riesgo para fuga anastomótica en casos de anastomosis colorrectal baja, principalmente, debido a los cambios anatómicos de la pelvis masculina, en un porcentaje de $18 \%$ comparado con $5 \%$ en el sexo femenino ${ }^{12}$. En el presente estudio, no se encontró que el sexo fuera un factor asociado a la aparición de fuga anastomótica $(\mathrm{p}=0,613)$.

En Colombia, en el 2015 y en un hospital de cuarto nivel de Bogotá, Neira determinó que los principales factores para desarrollar una fístula intestinal eran la presencia de peritonitis, seguida del antecedente de neoplasia maligna, la liberación de adherencias, la hipoalbuminemia y el uso de polipropileno como material de sutura ${ }^{18}$. En el presente estudio, se obtuvieron resultados similares; las variables más representativas fueron el antecedente de cirugía abdominal (76 \%) y la hipertensión arterial sistémica (33\%).

La hipoalbuminemia no representó una diferencia importante, ya que no se valoró en un gran número de pacientes, pues se utilizó la escala subjetiva de nutrición desarrollada por Detsky en $1987^{19}$. Se demostró que el estado nutricional y el material de sutura utilizado (especialmente, el polipropileno), eran factores asociados con la fístula anastomótica.

La falla anastomótica, que se manifiesta como fístula intestinal, tiene una incidencia variada según el segmento gastrointestinal intervenido: en las anastomosis gastroduodenales, hasta el $10 \%$ puede presentar falla ${ }^{34,7}$; en las anastomosis entre intestino delgado o entre intestino delgado y colon, puede ascender hasta $15 \%$, y en las anastomosis colorrectales, se pueden presentar hasta el $6,0 \%{ }^{3,4,8,9}$. Sin embargo, en el análisis de los presentes resultados no se documentó que el tipo de anastomosis, ni tampoco el segmento gastrointestinal intervenido, fueran factores asociados con la aparición de fístula anastomótica.

Un debate importante y que cada vez genera más controversia sobre los beneficios y las desventajas aportadas por las suturas mecánicas en el campo de la cirugía gastrointestinal, no puede escapar del ámbito de las fístulas anastomóticas. La revisión sistemática de Sanabria, et al., no muestra ningún beneficio sobre las suturas manuales de intestino delgado con intestino delgado, ni de colon con colon; tampoco muestra ninguna relevancia al comparar el uso de una línea de sutura con el de dos líneas de sutura ${ }^{20}$.

El resultado del presente estudio no es diferente a lo reportado en este estudio nacional; se encontró una frecuencia discretamente elevada (67\%) del uso de suturas mecánicas y, en los pacientes que presentaron fístula intestinal, el $62 \%$ de estas suturas fueron mecánicas, por lo cual se considera que son un factor asociado con esta complicación. La utilización de suturas mecánicas sigue en aumento pues reducen el tiempo quirúrgico; sin embargo, el incremento en los costos es uno de los impedimentos para su masificación en países en desarrollo, como Colombia.

Es pertinente mencionar que las fístulas fueron más frecuentes en cirugías de urgencia, 17 pacientes (81\%), 
lo cual es totalmente equiparable con las cifras mundiales, en las que el trauma desempeña un papel fundamental; por lo tanto, la cirugía de urgencia es otro factor asociado con la fístula anastomótica.

Aunque el principal objetivo del presente estudio fue calcular la incidencia de la fístula anastomótica, también es importante el tiempo de su aparición. El día séptimo del posoperatorio fue el de mayor frecuencia, lo que corresponde a los esperado según las características fisiopatológicas esperadas del desarrollo y la evolución de las fistulas ${ }^{3,12}$. Asimismo, la estancia hospitalaria pasa de una media de siete días cuando no se presentan fístulas, a una de 25 cuando se presenta esta complicación, como ocurrió en un paciente.

En todos los pacientes, el diagnóstico se hizo por las manifestaciones clínicas y los hallazgos quirúrgicos, y en ninguno se requirió de exámenes adicionales de imágenes.

En los pacientes sometidos a resección intestinal y anastomosis, sus condiciones previas y el comportamiento hemodinámico intraoperatorio fueron factores determinantes para el buen funcionamiento de la anastomosis. Por esto, cobra importancia el uso de vasopresores intraquirúrgicos, ya que estos generan un mecanismo compensatorio de vasoconstricción mesentérica, lo cual puede producir isquemia en la zona de la anastomosis ${ }^{3}$. En una tercera parte de los pacientes que presentaron fístula, se requirieron dosis intermedias de vasopresores, circunstancia que podría ser objeto de futuras investigaciones, ya que podría aumentar el riesgo de presentar fístula. En cuanto al comportamiento hemodinámico, en este estudio el sangrado intraoperatorio no demostró ser un factor asociado con la aparición de fístula anastomótica.

Si bien es cierto que la fístula intestinal es una complicación temida por el cirujano después de una anastomosis, también se presentan otras complicaciones en casos de resección intestinal y anastomosis. En 1992, Clavien, et al., clasificaron las complicaciones posquirúrgicas en cinco grados, según el comportamiento multiorgánico presentado ${ }^{21}$. La infección del sitio operatorio de tipo órgano y espacio, de grado II según esta clasificación, fue la más prevalente en este estudio (33\%), seguida de la evisceración y la obstrucción intestinal.

Como fortaleza en este estudio cabe resaltar que se contó con dos servicios de cirugía general, los cuales cuentan con un amplio grupo de cirujanos generales y subespecialistas reconocidos en el ámbito nacional e internacional; además, los criterios clínicos y las decisiones fueron estandarizadas según las técnicas quirúrgicas mundialmente utilizadas en centros de gran experiencia, lo cual permitió la correcta y oportuna atención de los pacientes incluidos en este proyecto. Sumado a esto, el llevar a cabo un estudio prospectivo en un ámbito quirúrgico a nivel nacional brinda una herramienta válida para medir la incidencia de fallas en las anastomosis, las cuales pueden servir como referencia para futuras publicaciones encaminadas a generar nuevas hipótesis sobre todos los mecanismos que influyen en este tipo de complicaciones.

Como limitación se considera la falta de oportunidad en la realización de los procedimientos dada la gran complejidad y el avanzado estado de deterioro de la mayoría de pacientes que ingresan a los servicios de urgencias, hecho que se vio reflejado en la mortalidad de nuestros pacientes que fueron llevados a anastomosis intestinal, 5,1\% del total de los pacientes que ingresaron al estudio y el 52,6 \% en la población que presentó el desenlace.

Un factor que se debe tener en cuenta en las anastomosis intestinales fue descrito por Nemeth, et al., en un estudio retrospectivo en el cual se evaluó la experiencia de los residentes de cirugía general en la práctica de suturas intestinales; en un periodo de entrenamiento de cinco años, cada uno practicó un promedio de 67,2 anastomosis, la mayoría ( $86 \%$ ) con sutura mecánica, obteniéndose un bajo porcentaje de complicaciones ${ }^{22}$.

Finalmente, como conclusiones del presente estudio, se puede afirmar que la incidencia de fístulas en las anastomosis fue la esperada según los reportes a nivel mundial, y que el antecedente de cirugía abdominal y la intervención quirúrgica de urgencia son los factores más relevantes para el desarrollo de fístula intestinal, sin desconocer que el éxito de la anastomosis también depende, en parte, de la experiencia del cirujano. 


\title{
Incidence of anastomotic failure in small bowel, colon and rectum, Bogotá, Colombia
}

\begin{abstract}
Introduction: Anastomotic leak is one of the main complications of patients submitted to gastrointestinal surgery. It occurs in approximately in 3.4\% to 15\% of cases. In Colombia the overall incidence of anastomotic leak is not known.

Material and methods: A prospective cohort study that included patients undergoing anastamosis and who were followed up for up to 30 days. A descriptive analysis of the information was performed. The variables were analyzed with the chi-square test and the Kaplan-Meller graphs were used to determine the time to the event. Data analyses was performed on STATA 13.

Results: 195 patients were admitted. 47.1\% were male, and the median age of the study group was 60 years (ICR: 48-70). The incidence of anastomotic failure was $10.8 \%$ and the incidence rate was 10.2 per 1000 person-years; $29.7 \%$ of the patients presented postoperative complications and a mortality rate of $9.7 \%$. The median hospital stay was 8 days (ICR: 5 - 16).

Conclusion: The incidence of anastomotic leak observed appears in accordance with reports published in the world literature. The history of abdominal surgery as well as emergency surgery were the most frequent factors among the patients who developed anastomosis leak.
\end{abstract}

Key words: Intestine, small; suture techniques; anastomosis, surgical; surgical wound dehiscence; intestinal fistula; protein-energy malnutrition.

\section{Referencias}

1. Booth CC. What has technology done to gastroenterology? Gut. 1985;26:1088-94

2. Bach SP, Mortensen NJ. Ileal pouch surgery for ulcerative colitis. World J Gastroenterol. 2007;13:3288-300.

3. Berry SM, Fischer JE. Classification and pathophysiology of enterocutaneous fistulas. Surg Clin North Am. 1996;76:1009-18.

4. Schecter WP. Management of enterocutaneous fistulas. Surg Clin North Am. 2011;91:481-91.

5. Tassiopoulos AK, Baum G, Halverson JD. Small bowel fistulas. Surg Clin North Am. 1996;76:1175-81.

6. Lavery IC. Colonic fistulas. Surg Clin North Am. 1996;76:118390.

7. Chung MA, Wanebo HJ. Surgical management and treatment of gastric and duodenal fistulas. Surg Clin North Am. 1996;76:1137-46.

8. Jung SH, Yu CS, Choi PW, Kim DD, Park IJ, Kim HC, et al. Risk factors and oncologic impact of anastomotic leakage after rectal cancer surgery. Dis Colon Rectum. 2008;51:902-8.

9. Girard E, Messager M, Sauvanet A, Benoist S, Piessen G, Mabrut JY, et al. Anastomotic leakage after gastrointestinal surgery: Diagnosis and management. J Visc Surg. 2014;151:441-50.

10. Bruce J, Krukowski ZH, Al-Khairy G, Russell EM, Park KG. Systematic review of the definition and measurement of anastomotic leak after gastrointestinal surgery. Br J Surg. 2001;88:1157-68.

11. Widdison AL. Proposed definitions for the audit of postoperative infection: A discussion paper. Ann R Coll Surg Engl. 1992;74:151-2.

12. Lipska MA, Bissett IP, Parry BR, Merrie AE. Anastomotic leakage after lower gastrointestinal anastomosis: Men are at a higher risk. ANZ J Surg. 2006;76:579-85.

13. Williams MD, Watts D, Fakhry S. Colon injury after blunt abdominal trauma: Results of the EAST Multi-Institutional Hollow Viscus Injury Study. J Trauma. 2003;55:906-12.

14. Brownstein MR, Bunting T, Meyer AA, Fakhry SM. Diagnosis and management of blunt small bowel injury: a survey of the membership of the American Association for the Surgery of Trauma. J Trauma. 2000;48:402-7.

15. Byrn JC, Schlager A, Divino CM, Weber KJ, Baril DT, Aufses $\mathrm{AH}$. The management of 38 anastomotic leaks after 1,684 intestinal resections. Dis Colon Rectum. 2006;49:1346-53.

16. Campos AC, Meguid MM, Coelho JC. Factors influencing outcome in patients with gastrointestinal fistula. Surg Clin North Am. 1996;76:1191-8.

17. Lévy E, Frileux P, Cugnenc PH, Honiger J, Ollivier JM, Parc R. High-output external fistulae of the small bowel: Management with continuous enteral nutrition. Br J Surg. 1989;76:676-9. 
18. Neira AM. Factores de riesgo para el desarrollo de fístulas gastrointestinales postoperatorias en hospital universitario mayor. Bogotá: Colegio Mayor Universidad del Rosario; 2015. Fecha de consulta: 15 de mayo de 2017. Disponible en: http://repository.urosario.edu.co/bitstream/handle/10336/10217/1032358734-2015.pdf?sequence=1.

19. Detsky AS, Baker JP, Mendelson RA, Wolman SL, Wesson DE, Jeejeebhoy KN. Evaluating the accuracy of nutritional assessment techniques applied to hospitalized patients: Methodology and comparisons. J Parenter Enteral Nutr. 1984;8:153-9.

20. Sanabria ÁE, Vega NV, Domínguez LC, Osorio C. Anastomosis intestinal: ¿manual o mecánica?, ¿en un plano o en dos planos? Revista Colombiana de Cirugía. 2010;25:97-103.
21. Clavien PA, Sanabria JR, Strasberg SM. Proposed classification of complications of surgery with examples of utility in cholecystectomy. Surgery. 1992;111:518-26.

22. Nemeth ZH, Lazar EL, Paglinco SR, Hicks AS, Lei J, BarrattStopper PA, et al. Experience of general surgery residents in the creation of small bowel and colon anastomoses. J Surg Educ. 2016;73:844-50.

Correspondencia: Maikel Adolfo Pacheco, MD

Correo electrónico: mailkel70@hotmail.com

Bogotá D.C., Colombia 